EPJ Web of Conferences 36, 00001 (2012)

DOI: $10.1051 /$ epjconf/20123600001

(C) Owned by the authors, published by EDP Sciences, 2012

\title{
Preface
}

\author{
A. Badalà ${ }^{1}$, M. Bleicher ${ }^{2}$, L. Fabbietti ${ }^{3,4}$, C. Markert ${ }^{5}$, R. F. Rapp ${ }^{6}$, J. Stroth $^{7}$ \\ ${ }^{1}$ INFN Sezione di Catania, V. S. Sofia 64, 95123, Catania, Italy \\ ${ }^{2}$ Frankfurt Institute for Advanced Studies, Ruth-Moufang-Str. 1, 60438 Frankfurt, Germany \\ ${ }^{3}$ Excellence Cluster 'Origin and Structure of the Universe', 85748, Garching, Germany \\ ${ }^{4}$ Physik Department E12, Technische Universität München, 85748, Garching, Germany \\ ${ }^{5}$ University of Texas at Austin, Physics Department, 1 University Station, C1600, Austin, TX 7812- \\ 0264, USA \\ ${ }^{6}$ Cyclotron Institute and Department of Physics and Astronomy, Texas A\&M University, College \\ Station, TX 77843-3366, USA \\ ${ }^{7}$ Institut für Kernphysik Goethe-Universität, 60438, Frankfurt, Germany
}

The first international workshop on hadronic resonances was held at the University of Texas at Austin in Austin Texas during 5-7 March 2012.

Many hadronic resonances are measured in heavy ion and elementary collisions at various energies. The question is still how all this results fit into a consistent picture within the signatures of chiral symmetry restoration in a medium near a transition from partonic matter evolving into hadronic matter. We felt that it is time to have an intended discussion between the experimentalist and the theorist over the full collision energy range and different collision species. The workshop was a great success and we left with a better understanding of the results from other experiments, the pros and cons of the theoretical models and the challenge to extract a signature of a modified medium. The workshop benefit mostly from the open, honest and critical discussion of all the participants. All questions where ask never the less how "painful" the answer might be. We would like to thank everyone contributing to the success of this workshop. And we hope to stay in touch till we meet again in 2-3 years for our second workshop to discuss our progress and new results. We also would like to thanks the local organizing committee and the University of Texas at Austin for their support and the smooth operation to make the stay in Austin a pleasant experience. In addition we would like to thank Dipartimento di Fisica e Astronomia, Università di Catania and the University of Texas at Austin for their financial support.

\section{Organizing Committee}

- Angela Badalà (INFN Sezione di Catania, Catania, Italy)

- Marcus Bleicher (Frankfurt Institute for Advanced Studies, Frankfurt, Germany)

- Laura Fabbietti (Technische Universität München, Garching, Germany)

- Thomas Hemmick (Stony Brook University)

- Jerry Hoffmann(University of Texas at Austin, Texas, USA)

- Christina Markert (University of Texas at Austin, Texas, USA)

- $\quad$ Ralph Friedrich Rapp ( Texas A\&M University, Texas, USA)

- Joachim Stroth (Institut für Kernphysik Goethe-Universität, Frankfurt, Germany)

This is an Open Access article distributed under the terms of the Creative Commons Attribution License 2.0, which permits unrestricted use, distribution, and reproduction in any medium, provided the original work is properly cited. 


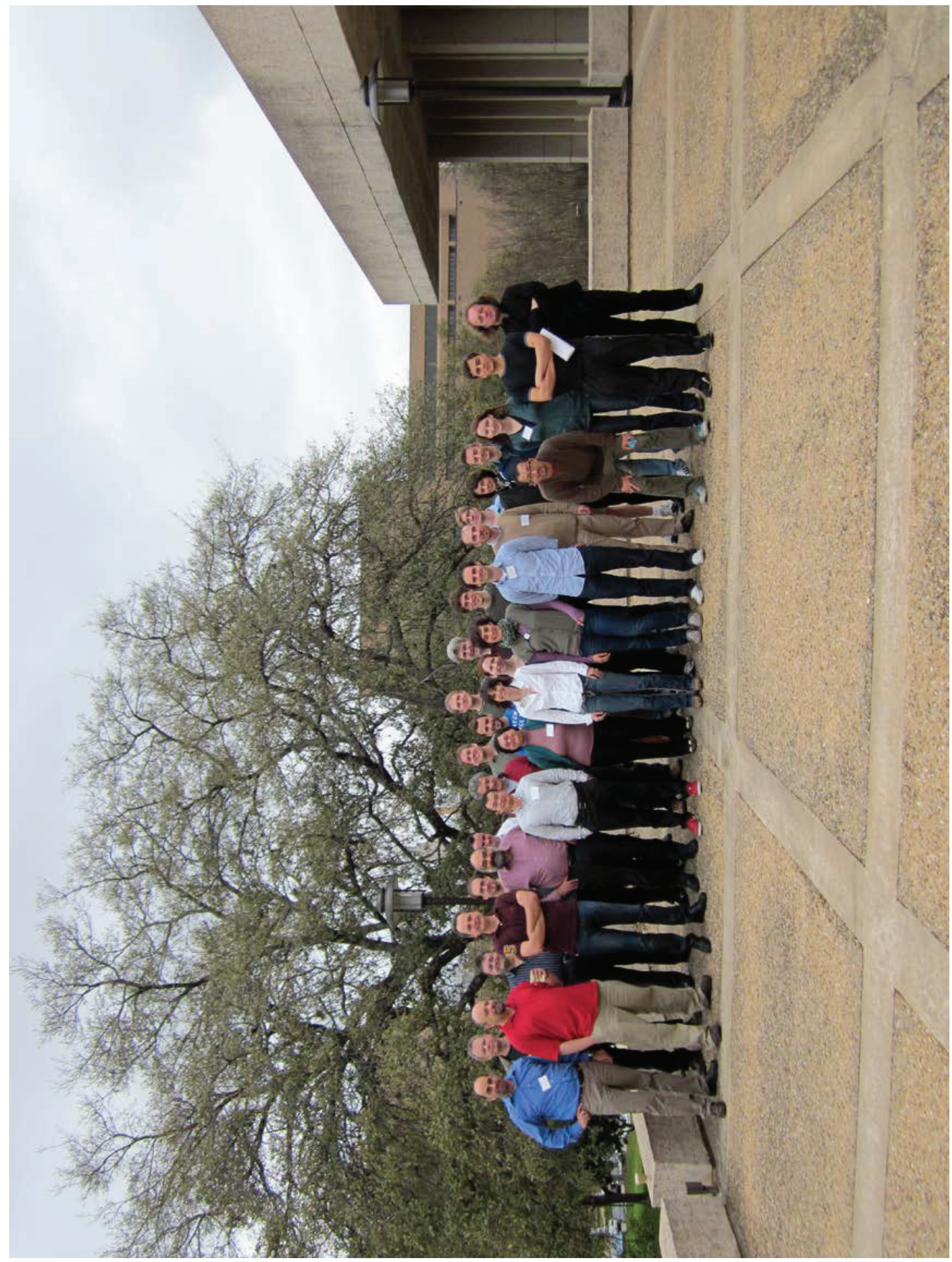




\section{Registered participant}

\begin{tabular}{lll} 
Joeg & Aichelin & Subatech \\
Angela & Badalà & INFN-Sezione di Catania \\
Christoph & Baumann & Goethe-Universität Frankfurt \\
Prabhat & Bhattarai & University of Texas at Austin \\
Markus & Bleicher & ITP \\
Elena & Bratkovskaya & FIAS\&ITP,University Frankfurt \\
Oliver & Busch & PI Heilderg \\
Sarah & Campbell & Iowa State University \\
Alan & Davila & University of Texas at Austin \\
Alessandro & De Falco & Università-INFN Cagliari \\
Heng-Tong & Ding & Brookhaven National Laboratory \\
Laura & Fabbietti & TU Munich \\
Enrico & Fragiacomo & INFN- Sezione di Trieste \\
Frank & Geurts & Rice University \\
Michael & Hartmann & Forschungszentrum Jülich \\
Jerry & Hoffmann & University of Texas at Austin \\
Paul & Hohler & Texas A\&M University \\
Anders & Knospe & University of Texas at Austin \\
Che-Ming & Ko & Texas A\&M University \\
Volker & Koch & LBNL \\
Manuel & Lorenz & Goethe-Universität Frankfurt \\
Christina & Markert & University of Texas at Austin \\
Michael & Murray & University of Kansas \\
Elizabeth & Oldag & University of Texas at Austin \\
Ralf & Rapp & Texas A\&M University \\
Lanny & Ray & University of Texas at Austin \\
Steffen & Strauch & University of South Carolina \\
Giorgio & Torrieri & FIAS, JW Goethe-Universität Frankfurt \\
Hendrik & van Hees & Goethe-Universität Frankfurt \\
Ivan & Vitev & LANL \\
Sasha & Vogel & FIAS, HGS-HIRe \\
Masayuki & Wada & University of Texas at Austin \\
Leonidas & Xaplanteris & University of Texas at Austin \\
& & \\
\hline
\end{tabular}

